\title{
THE EFFECT OF ISOTOPIC SPLITTING ON THE BISECTOR AND INVERSIONS OF THE SOLAR Ca II 854.2 nm LINE
}

\author{
Jorrit LeenaArts ${ }^{1,2}$, Jaime de la Cruz Rodríguez ${ }^{2}$, Oleg Kochukhov ${ }^{3}$, and Mats Carlsson ${ }^{1}$ \\ ${ }^{1}$ Institute of Theoretical Astrophysics, University of Oslo, P.O. Box 1029 Blindern, N-0315 Oslo, Norway; jorritl@astro.uio.no, mats.carlsson@astro.uio.no \\ ${ }^{2}$ Institute for Solar Physics, Department of Astronomy, Stockholm University, AlbaNova University Centre, SE-106 91 Stockholm Sweden; jaime@ astro.su.se \\ ${ }^{3}$ Department of Physics and Astronomy, Uppsala University, Box 516, SE-75120 Uppsala, Sweden; oleg.kochukhov@physics.uu.se \\ Received 2014 January 20; accepted 2014 February 27; published 2014 March 10
}

\begin{abstract}
The Ca II $854.2 \mathrm{~nm}$ spectral line is a common diagnostic of the solar chromosphere. The average line profile shows an asymmetric core, and its bisector shows a characteristic inverse-C shape. The line actually consists of six components with slightly different wavelengths depending on the isotope of calcium. This isotopic splitting of the line has been taken into account in studies of non-solar stars, but never for the Sun. We performed non-LTE radiative transfer computations from three models of the solar atmosphere and show that the line-core asymmetry and inverse C-shape of the bisector of the $854.2 \mathrm{~nm}$ line can be explained by isotopic splitting. We confirm this finding by analyzing observations and showing that the line asymmetry is present irrespective of conditions in the solar atmosphere. Finally, we show that inversions based on the Ca II $854.2 \mathrm{~nm}$ line should take the isotopic splitting into account, otherwise the inferred atmospheres will contain erroneous velocity gradients and temperatures.
\end{abstract}

Key words: radiative transfer - Sun: atmosphere - Sun: chromosphere

Online-only material: color figures

\section{INTRODUCTION}

The triplet of lines of CaII at 849.8, 854.2 and $866.2 \mathrm{~nm}$ in the solar spectrum are formed in the chromosphere of the Sun. They are common diagnostics of the chromosphere. The infrared triplet is sensitive to magnetic fields and is used to infer properties of the chromospheric magnetic field based on observations of the Stokes vector (e.g., Socas-Navarro et al. 2000a, 2006; López Ariste et al. 2001; Judge et al. 2010; de la Cruz Rodríguez \& Socas-Navarro 2011).

All three lines show a marked asymmetry in their line cores in spatially and temporally averaged profiles (Neckel \& Labs 1984), and their bisector shows an inverse-C shape (Uitenbroek 2006). The $\mathrm{H} \alpha$ line, which forms at similar heights in the chromosphere, does not show such asymmetry (Chae et al. 2013).

The variation of the bisector of the Ca II $854.2 \mathrm{~nm}$ line during the solar cycle has been investigated by Pietarila \& Livingston (2011), who found that the asymmetry of the line core, and thus the bisector, changes in phase with the solar magnetic cycle. In a follow-up study, Pietarila \& Harvey (2013) found that the bisector shape in areas with high magnetic flux is different from the bisector in the quiet Sun, providing a natural explanation for the asymmetry-activity correlation.

The actual physical mechanism that causes the solar line asymmetry has so far not been identified. Previous modeling efforts did not reproduce the observed inverse-C-shaped bisector (Uitenbroek 2006; Leenaarts et al. 2009). These studies ignored the minority isotopes of calcium and instead assumed all calcium is in the form of ${ }^{40} \mathrm{Ca}$, the most abundant isotope $(96.94 \%$ in the standard solar system composition; Anders \& Grevesse 1989)

In the stellar community it has however been realized that the other isotopes should be taken into account in order to explain the observed line shapes in chemically peculiar stars (e.g., Castelli \& Hubrig 2004; Cowley \& Hubrig 2005; Cowley et al. 2007; Ryabchikova et al. 2008).

With this Letter we draw the attention of the solar community to the importance of isotopic splitting in the modeling of the Ca II
IR triplet. Using appropriate chromospheric models, we show that isotopic splitting can explain the observed inverse-C-shaped bisector of the Ca IR triplet lines. In addition we demonstrate that neglecting the splitting in inversions of observed Stokes profiles can lead to erroneous derivation of the velocity, temperature, and possibly, magnetic field strength.

\section{SIMULATIONS AND RADIATIVE TRANSFER}

We constructed five-level-plus-continuum model atoms for the four stable isotopes and two extremely long-lived isotopes of calcium. We computed the energy levels of the $4 p^{2} P_{3 / 2,5 / 2}$ and $3 d^{2} D_{1 / 2,3 / 2}$ states using the experimental data from MårtenssonPendrill et al. (1992) and Nörtershäuser et al. (1998). There is a near-linear relation between the number of nucleons and the wavelength of each line in the IR triplet; we used this relation to extrapolate the energy levels for isotopes that were not measured. One isotope, ${ }^{43} \mathrm{Ca}$, shows hyperfine splitting (Safronova \& Safronova 2011). We do not take this into account. Because the spread of this splitting is only $0.88 \mathrm{pm}$, this does not significantly influence our results.

We took the total calcium abundance from Asplund et al. (2009) and the relative abundance of each isotope from Anders \& Grevesse (1989). In Table 1 we give the isotopes, their abundance in the standard $A_{\mathrm{H}}=12$ scale and the wavelength of the Ca II $854.2 \mathrm{~nm}$ line.

We used three model atmospheres. The first is the 1D semiempirical FAL model C of Fontenla et al. (1993). The second is a time series of 240 snapshots taken at $10 \mathrm{~s}$ intervals computed with the 1D radiation-hydrodynamics code RADYN (e.g., Carlsson \& Stein 1992, 1997). The third is a snapshot of a 3D radiation-MHD simulation performed with the Bifrost code (Gudiksen et al. 2011). We chose the same snapshot as was used by Leenaarts et al. (2012) to investigate $\mathrm{H} \alpha$ line formation, and refer to that paper for details of the simulation.

We performed the non-LTE radiative transfer computations using the RH code by Uitenbroek (2001). For each atmospheric 
Table 1

Ca Isotopes, Their Abundance and $3 d^{2} D_{5 / 2}-4 p^{2} P_{3 / 2}$ Line Wavelengths

\begin{tabular}{lcc}
\hline \hline Isotope & Abundance & $\begin{array}{c}\lambda \\
(\mathrm{nm})\end{array}$ \\
\hline${ }^{40} \mathrm{Ca}$ & 6.33 & 854.20857 \\
${ }^{42} \mathrm{Ca}$ & 4.15 & 854.21426 \\
${ }^{43} \mathrm{Ca}$ & 3.47 & 854.21696 \\
${ }^{44} \mathrm{Ca}$ & 4.66 & 854.21952 \\
${ }^{46} \mathrm{Ca}$ & 1.94 & 854.22433 \\
${ }^{48} \mathrm{Ca}$ & 3.61 & 854.22871 \\
\hline
\end{tabular}

model we performed two computations: one where we assumed all calcium to be in the form of ${ }^{40} \mathrm{Ca}$, and one where we included all isotopes given in Table 1. In the multi-isotope computation we included the isotopes as separate atoms that were treated simultaneously in non-LTE. FALC included a microturbulence that varies with height as given in Vernazza et al. (1981). In the RADYN simulation we used a constant microturbulence of $2 \mathrm{~km} \mathrm{~s}^{-1}$. The 3D Bifrost snapshot did not include microturbulence. Each column in this model was treated independently as a 1D plane-parallel atmosphere.

\section{RESULTS}

\subsection{Profile Asymmetry and Bisector}

In Figure 1 we show the vertically emergent profiles of the Ca II $854.2 \mathrm{~nm}$ line from our model atmospheres with and without isotopic splitting and compare the to the observed quietSun atlas profile from Neckel \& Labs (1984). The profiles for the RADYN simulation are time averages. The Bifrost profiles are spatial averages over the simulation box.

The synthetic profiles appear all nearly symmetric when isotopic splitting is ignored, and are narrower than the atlas. With the inclusion of the isotopic splitting the profiles become wider and asymmetric, also in the case of the static FALC atmosphere. The FALC profile only show asymmetry in the upper part of the line core. This lack of deep-core asymmetry is caused by the chromospheric temperature rise that masks the presence of the heavier isotopes. The RADYN and Bifrost profiles show a modest asymmetry that is present in the whole line core. None of the models reproduces the observed core width or intensity. The too small core width indicates insufficiently strong vertical motions (cf. Leenaarts et al. 2009), the too low core intensity is mainly caused by a too low temperature in the model chromospheres.

Inspection of the 849.8 and $866.2 \mathrm{~nm}$ lines shows that those lines behave the same: the line cores are nearly symmetric without isotopic splitting but show a strong asymmetry when the splitting is included.

We computed the bisector of all line profiles. We define the bisector $b(I)$ as function of the intensity $I$ as

$$
b(I)=\frac{1}{2}\left(\lambda_{\text {red }}(I)+\lambda_{\text {blue }}(I)\right)-\lambda_{\min },
$$

with $\lambda_{\text {red }}(I)$ the wavelength on the red side of the profile minimum where the intensity is $I$, and similarly for $\lambda_{\text {blue }}(I)$, the quantity $\lambda_{\min }$ is the wavelength of the profile minimum. The bisector is by construction only sensitive to the line asymmetry, and not to the line width. By defining the zero point to be the profile minimum we allow direct comparison of the bisector amplitude and avoid uncertainties in the absolute wavelength calibration of the atlas profile.
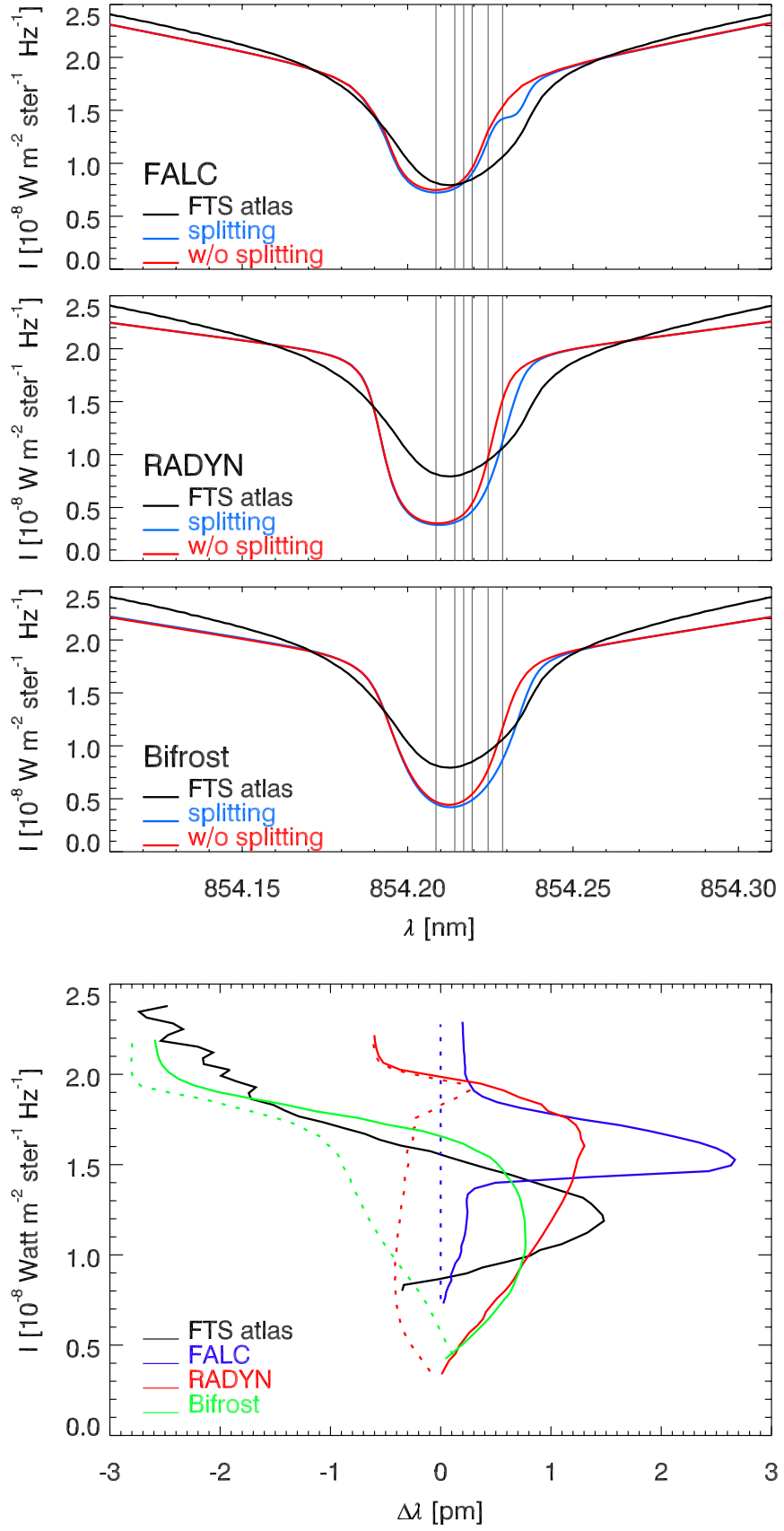

Figure 1. Upper three panels: comparison of the observed atlas profile (black) of the Ca II $854.2 \mathrm{~nm}$ line with synthetic profiles computed from three different atmosphere models with isotopic splitting (blue) and without splitting (red). The vertical grey lines indicate the wavelength of the transition for each isotope (see Table 1). Bottom panel: bisectors of the Ca II $854.2 \mathrm{~nm}$ line as determined from the atlas profile and the synthetic profiles from the atmosphere models. Solid curves are with splitting, dotted curves without splitting. The zero point of the $\Delta \lambda$-scale is the wavelength of the minimum intensity of each line profile.

(A color version of this figure is available in the online journal.)

We display the resulting bisectors in the bottom panel of Figure 1. The atlas profile shows the observed quiet-Sun inverse$\mathrm{C}$ shape. The models do not show this shape when splitting is ignored. Note that we obtain the same bisector for the RADYN model, ignoring splitting as in Uitenbroek (2006).

This inverse-C shape is qualitatively reproduced by the models when isotopic splitting is included. FALC has nearzero amplitude in the deep core and a sudden increase of the amplitude in the upper core beyond the observed amplitude. 
It does not display the turn toward the blue at larger intensity as in the atlas profile. RADYN exhibits the correct bisector amplitude, but not the turn toward the blue. The Bifrost model does not reach the observed bisector amplitude, but reproduces the blueward turn. As Bifrost is the only model with a convection zone, we speculate that this blueward turn is caused by overturning convection in the upper photosphere.

\subsection{Observations}

To support our theoretical results, we explored a Ca II $854.2 \mathrm{~nm}$ dataset acquired at the Swedish 1-m Solar Telescope (SST) with the CRISP instrument. The images were taken at solar disk center in the quiet Sun on the 2013 July 14 at 10:38 UT. The line was observed with a sampling of $5.6 \mathrm{pm}$ in the line core up to $\Delta \lambda= \pm 42 \mathrm{pm}$. In the wings, the sampling was coarser. At $854.2 \mathrm{~nm}$, CRISP has a spectral resolution of $\delta \lambda=11.1 \mathrm{pm}$, thus our profiles are critically sampled close to line center. The data have been processed as described in de la Cruz Rodríguez et al. (2013).

Hole (2012) analyzed a similar dataset, trying to associate the inverse-C-shaped bisector with specific solar features or dynamics. The author detected the inverse-C shape in spatially resolved profiles, and concluded the bisector shape is thus not an effect of averaging different profiles that individually do not show an inverse-C-shaped bisector.

We divided the field of view in our data in three regions: bright points, chromospheric fibrils, and the rest of the quiet Sun. Then we computed spatially averaged line profiles within each region, and calculated the bisectors as shown in Figure 2. We find that inverse-C-shaped bisectors are present in spectra from fibrils, bright points, and quiet Sun, but only the quiet-Sun bisector shows the blueward turn at higher intensities. The bisector amplitudes in each region are of the same order of magnitude to that from the Fourier transform spectroscope (FTS) atlas. Isotopic splitting naturally explains the ubiquitous presence of the asymmetry, despite the variation in thermodynamical properties.

Note that the bisector amplitude is larger in fibrils and bright points than in the quiet Sun, and the blueward turn is suppressed. This is in agreement with Pietarila \& Harvey (2013) who found the same behavior in circumfacular regions compared to the quiet Sun.

\subsection{Influence on Inversions}

Inversions of solar observations in the Ca II $854.2 \mathrm{~nm}$ line have so far ignored isotopic splitting (e.g., Tziotziou et al. 2001; Pietarila et al. 2007; de la Cruz Rodríguez et al. 2013). The additional line width and asymmetry caused by the minority isotopes will then typically be fitted with a velocity gradient. We investigated this effect by inverting the synthetic full Stokes vector including isotopic splitting computed from a column from the Bifrost simulation taken from a magnetic element. In order to mimic real observing conditions we convolved the profile with the CRISP spectral resolution and resampled it at the CRISP critical sampling of $5.6 \mathrm{pm}$.

The inversions were performed with the NICOLE code in non-LTE (Socas-Navarro et al. 2000b), using an inversion strategy similar to de la Cruz Rodríguez et al. (2012). NICOLE iteratively modifies the physical parameters in a $1 \mathrm{D}$ model atmosphere to reproduce observed full-Stokes profiles.

The inversion was performed twice. Once assuming all calcium is ${ }^{40} \mathrm{Ca}$, and once using an approximation to include overlapping lines by Carlsson (1986). In this approximation the Ca II $854.2 \mathrm{~nm}$ absorption coefficient is a weighted sum of Voigt
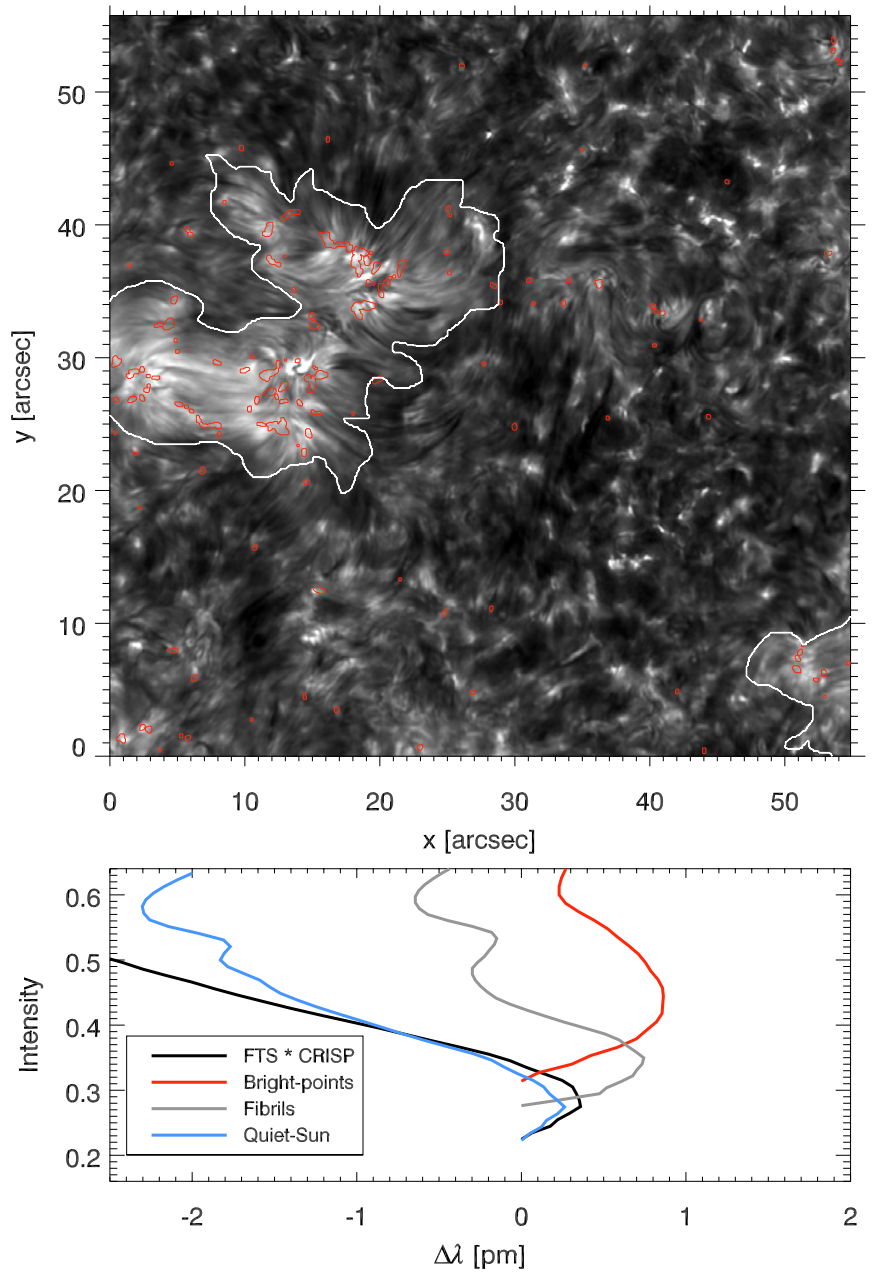

Figure 2. SST observations in the Ca II $854.2 \mathrm{~nm}$ line. Top: line core image. Masks enclosing bright points (red) and fibrils (white) are indicated with contours. Bottom: bisectors computed from spatially averaged line profiles, using the masks indicated in the top panel, for fibrils (gray), quiet Sun (blue), and bright points (red). The bisector from the FTS atlas convolved with the CRISP spectral profile is shown in black.

(A color version of this figure is available in the online journal.)

profiles, each centered at the rest wavelength of the line for each isotope. The weight is proportional to the relative abundance of each isotope. This is an approximation because the ratio of the level populations between different isotopes is not constant in the atmosphere. This method is not as accurate as treating each isotope separately, but it is much faster, and test calculations show that the differences in the resulting line profiles are insignificant. The inversion employed 11 temperature nodes and 4 nodes for the vertical velocity and 3 nodes for the vertical magnetic field. The results are shown in Figure 3.

The inversion including isotopic splitting recovers Stokes $I$ and the atmospheric parameters well, but it does not quite fit the amplitude of the Stokes $V$ peak on the red side of the line core.

If isotopic splitting is ignored, both Stokes $I$ and $V$ are fitted slightly worse. In order to fit the profile asymmetry, the inversion without splitting introduces a velocity field with up to $6 \mathrm{~km} \mathrm{~s}^{-1}$ difference to the true value and adjusts the temperature up to $2 \mathrm{kK}$. The resulting inferred atmosphere does not resemble the original atmosphere.

The Zeeman response in the Ca II $854.2 \mathrm{~nm}$ line is usually in the weak-field regime $(B<2500 \mathrm{G}$; see de la Cruz Rodríguez et al. 2013). Therefore, if the inversion is able to fit Stokes $I$, then 

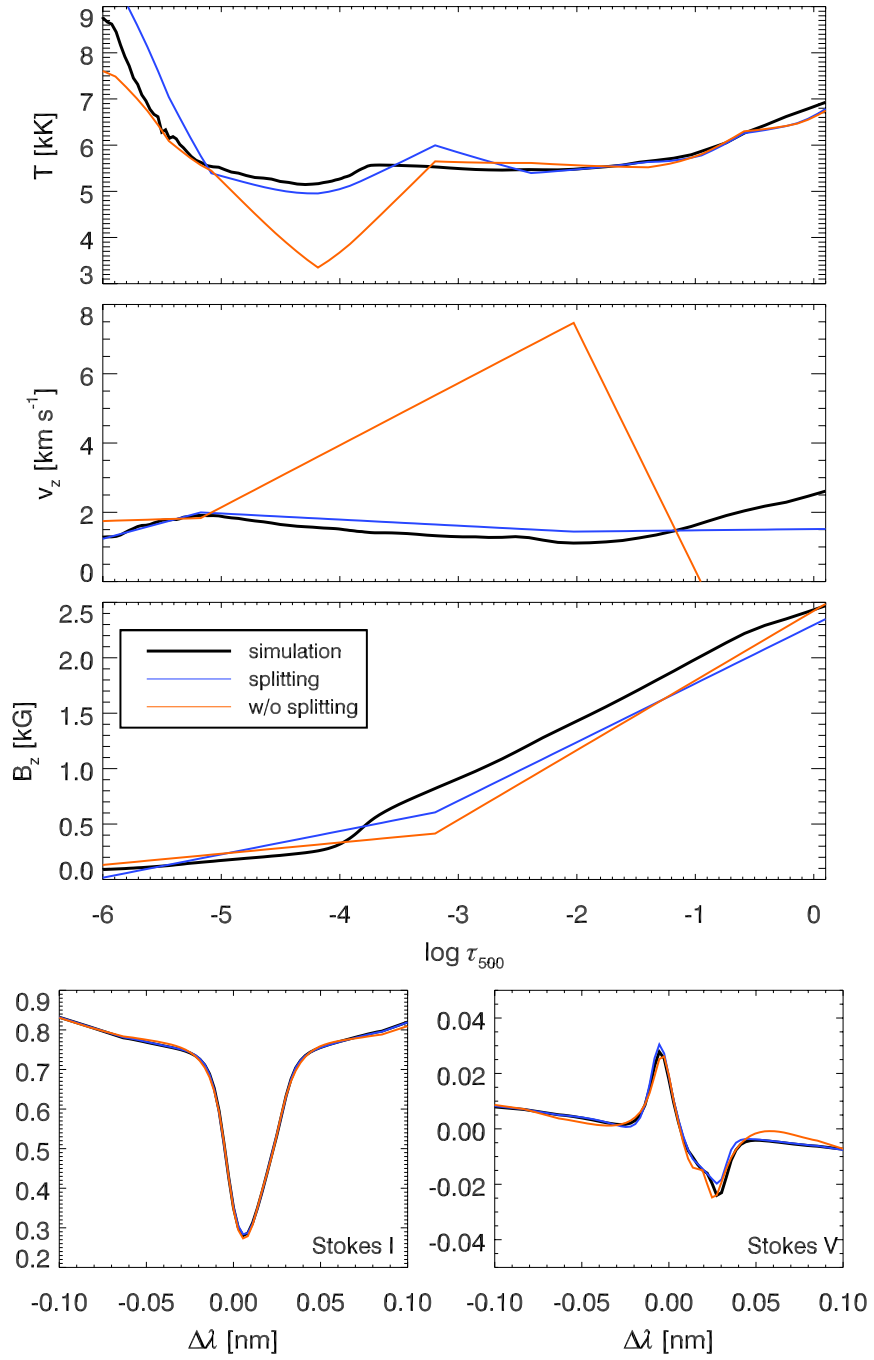

Figure 3. Comparison between the original Bifrost atmosphere (black) and the inverted quantities including isotopic splitting (blue) and without splitting (red). The three top rows show temperature, vertical velocity, and the vertical magnetic field. The bottom row shows the fits for Stokes $I$ and $V$ using the same color coding.

(A color version of this figure is available in the online journal.)

the magnetic field is retrieved accurately irrespective of whether isotopic splitting is included in the inversion. This is the case in our computation. But if the inversion cannot reproduce Stokes $I$, errors can also appear in the inferred magnetic field.

We also inverted the intensity profile from a row of pixels from the observations (512 in total) using seven nodes in temperature and four nodes in vertical velocity. These profiles were also inverted twice: with and without isotopic splitting. Figure 4 shows the results for a pixel with a clearly asymmetric line profile. The line profile is fitted equally well with both methods. However, the inversion without splitting introduces a velocity field in order to fit the line shape, similar to the inversion of the synthetic profile. Both inversions provide very similar estimates for the temperature as a function of height, in contrast to the synthetic data. The difference in inferred velocity structure is common: $62 \%$ of the pixels we inverted with and without splitting show a difference larger than $2 \mathrm{~km} \mathrm{~s}^{-1}$.

Finally, we note that the inversion without splitting needs four velocity nodes in order to fit the profile. The inversion including splitting reaches a good fit using only two nodes.
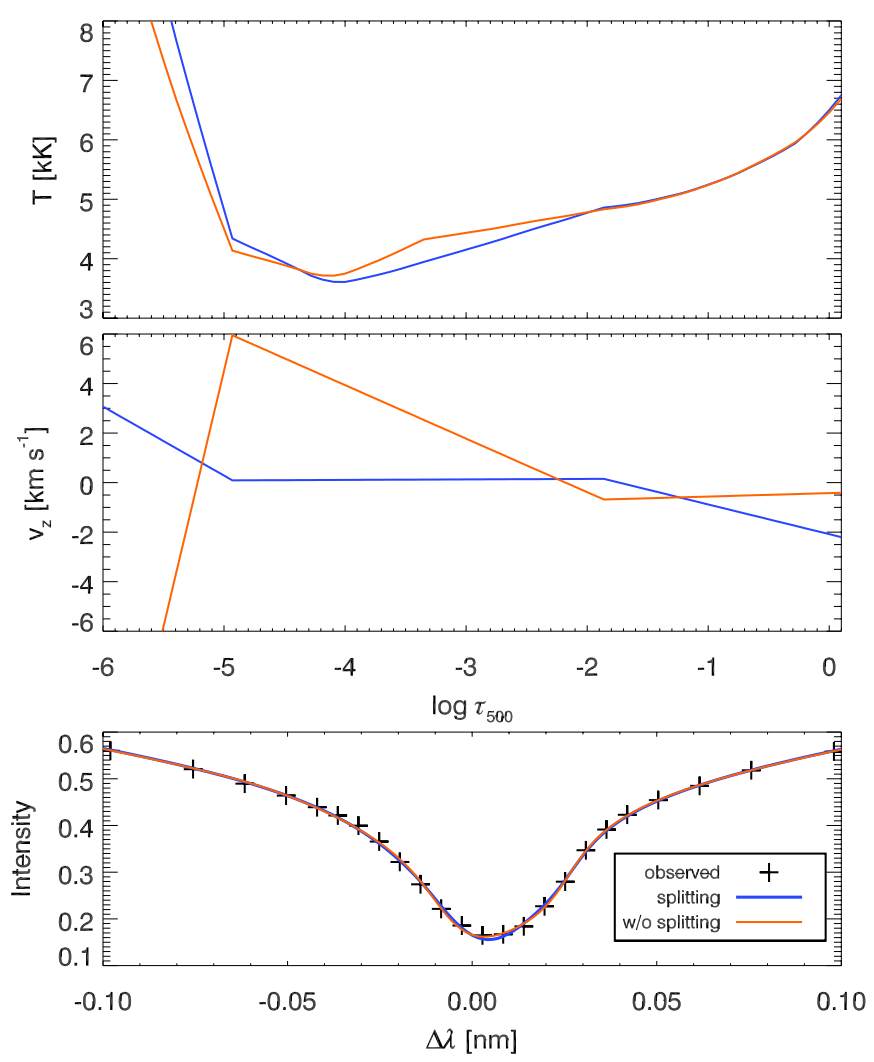

Figure 4. Inversion of an observed quiet-Sun profile from our observations, including isotopic splitting (blue) and without isotopic splitting (red). From top to bottom, the panels show temperature, line-of-sight velocity, and the line profile. The observed profile is indicated with black crosses in the bottom panel. (A color version of this figure is available in the online journal.)

\section{DISCUSSION AND CONCLUSIONS}

The various isotopes of Ca have slightly different energies for the $3 d$ and $4 p$ levels of Ca II. This results in isotopic splitting of the infrared triplet lines. This is of importance because the Ca II $854.2 \mathrm{~nm}$ line is an often-used diagnostic of the solar chromosphere.

We investigated this splitting in a static 1D and dynamic $1 \mathrm{D}$ and 3D models of the solar atmosphere. All employed models produce an asymmetric line core with an inverse-Cshaped bisector. These effects are not present when ignoring the minority isotopes. No model reproduces the observed quiet-Sun spectrum quantitatively, but all models agree qualitatively. We therefore conclude that the Ca II $854.2 \mathrm{~nm}$ line asymmetry and bisector shape is mainly caused by isotopic splitting and not by effects caused by velocity fields as has been suggested earlier (Uitenbroek 2006).

The exact bisector shape and amplitude in our models depend on the atmospheric structure. In our observations we find a markedly different bisector shape between the quiet Sun and regions with stronger magnetic field, although both show the inverse-C shape. This agrees with the findings of Pietarila \& Harvey (2013) and the variation of the bisector shape with the solar cycle (Pietarila \& Livingston 2011).

In addition we investigated the effect of isotopic splitting on the inversion of line profiles. We found that ignoring isotopic splitting in a test inversion of a synthetic line profile leads to an inferred atmospheric structure that contains spurious velocity gradients and an incorrect temperature variation in order to fit the line-core asymmetry. Inversions of observed line profiles 
indicate similar differences in inferred velocities with and without the inclusion of isotopic splitting. Modeling splitting by adding the line profiles of the different isotopes together largely eliminates these biases, without additional computational cost.

We recommend that all future inversions of the Ca II infrared lines include the effects of isotopic splitting.

This research was supported by the Research Council of Norway through the grant "Solar Atmospheric Modeling," from the European Research Council under the European Union's Seventh Framework Programme (FP7/2007-2013)/ ERC grant agreement No. 291058, and through grants of computing time from the Programme for Supercomputing of the Research Council of Norway. The Swedish 1-m Solar Telescope is operated on the island of La Palma by the Institute for Solar Physics of Stockholm University in the Spanish Observatorio del Roque de los Muchachos of the Instituto de Astrofsica de Canarias. We thank Luc Rouppe van der Voort for illuminating discussions and Andrew McWilliam for pointing out the hyperfine splitting of ${ }^{43} \mathrm{Ca}$.

\section{REFERENCES}

Anders, E., \& Grevesse, N. 1989, GeCoA, 53, 197

Asplund, M., Grevesse, N., Sauval, A. J., \& Scott, P. 2009, ARA\&A, 47, 481 Carlsson, M. 1986, UppOR, 33

Carlsson, M., \& Stein, R. F. 1992, ApJL, 397, L59

Carlsson, M., \& Stein, R. F. 1997, ApJ, 481, 500

Castelli, F., \& Hubrig, S. 2004, A\&A, 421, L1
Chae, J., Park, H.-M., Ahn, K., et al. 2013, SoPh, 288, 89

Cowley, C. R., \& Hubrig, S. 2005, A\&A, 432, L21

Cowley, C. R., Hubrig, S., Castelli, F., González, J. F., \& Wolff, B. 2007, MNRAS, 377, 1579

de la Cruz Rodríguez, J., Rouppe van der Voort, L., Socas-Navarro, H., \& van Noort, M. 2013, A\&A, 556, A115

de la Cruz Rodríguez, J., \& Socas-Navarro, H. 2011, A\&A, 527, L8

de la Cruz Rodríguez, J., Socas-Navarro, H., Carlsson, M., \& Leenaarts, J. 2012, A\&A, 543, A34

Fontenla, J. M., Avrett, E. H., \& Loeser, R. 1993, ApJ, 406, 319

Gudiksen, B. V., Carlsson, M., Hansteen, V. H., et al. 2011, A\&A, 531, A154

Hole, I. B. A. 2012, Master's thesis, University of Oslo

Judge, P. G., Tritschler, A., Uitenbroek, H., et al. 2010, ApJ, 710, 1486

Leenaarts, J., Carlsson, M., Hansteen, V., \& Rouppe van der Voort, L. 2009, ApJL, 694, L128

Leenaarts, J., Carlsson, M., \& Rouppe van der Voort, L. 2012, ApJ, 749, 136

López Ariste, A., Socas-Navarro, H., \& Molodij, G. 2001, ApJ, 552, 871

Mårtensson-Pendrill, A.-M., Ynnerman, A., Warston, H., et al. 1992, PhRvA, 45,4675

Neckel, H., \& Labs, D. 1984, SoPh, 90, 205

Nörtershäuser, W., Blaum, K., Icker, K., et al. 1998, EPJD, 2, 33

Pietarila, A., \& Harvey, J. W. 2013, ApJ, 764, 153

Pietarila, A., \& Livingston, W. 2011, ApJ, 736, 114

Pietarila, A., Socas-Navarro, H., \& Bogdan, T. 2007, ApJ, 670, 885

Ryabchikova, T., Kochukhov, O., \& Bagnulo, S. 2008, A\&A, 480, 811

Safronova, M. S., \& Safronova, U. I. 2011, PhRvA, 83, 012503

Socas-Navarro, H., Elmore, D., Pietarila, A., et al. 2006, SoPh, 235, 55

Socas-Navarro, H., Trujillo Bueno, J., \& Ruiz Cobo, B. 2000a, Sci, 288, 1396

Socas-Navarro, H., Trujillo Bueno, J., \& Ruiz Cobo, B. 2000b, ApJ, 530, 977

Tziotziou, K., Heinzel, P., Mein, P., \& Mein, N. 2001, A\&A, 366, 686

Uitenbroek, H. 2001, ApJ, 557, 389

Uitenbroek, H. 2006, ApJ, 639, 516

Vernazza, J. E., Avrett, E. H., \& Loeser, R. 1981, ApJS, 45, 635 